\title{
REVIEW ARTICLE Surfactant replacement therapy: from biological basis to current clinical practice
}

\author{
Roland Hentschel $\mathbb{D}^{1}$, Kajsa Bohlin ${ }^{2}$, Anton van Kaam ${ }^{3}$, Hans Fuchs ${ }^{1}$ and Olivier Danhaive ${ }^{4,5}$
}

This review summarizes the current knowledge on the physiological action of endogenous and exogenous pulmonary surfactant, the role of different types of animal-derived and synthetic surfactants for RDS therapy, different modes of administration, potential risks and strategies of ventilation, and highlights the most promising aims for future development. Scientists have clarified the physicochemical properties and functions of the different components of surfactant, and part of this successful research is derived from the characterization of genetic diseases affecting surfactant composition or function. Knowledge from functional tests of surfactant action, its immunochemistry, kinetics and homeostasis are important also for improving therapy with animal-derived surfactant preparations and for the development of modified surfactants. In the past decade newly designed artificial surfactants and additives have gained much attention and have proven different advantages, but their particular role still has to be defined. For clinical practice, alternative administration techniques as well as postsurfactant ventilation modes, taking into account alterations in lung mechanics after surfactant placement, may be important in optimizing the potential of this most important drug in neonatology.

Pediatric Research (2020) 88:176-183; https://doi.org/10.1038/s41390-020-0750-8

\section{INTRODUCTION}

Pulmonary surfactant, discovered in the 1950s, has been studied from the 1980s on to treat and prevent respiratory distress syndrome (RDS) in preterm infants. At that time manufacturing of surfactant preparations from porcine and bovine origin started, paralleled by a stunning increase in controlled trials within a few years. Administration of exogenous surfactant to preterm infants with RDS started its triumphal procession rapidly across many countries, hindered only by relatively high costs of therapy. Numerous subsequent trials in the past decades confirmed not only the effectiveness of surfactant to improve mortality, need for ventilation and short-term outcome, but also to have a huge impact on residual morbidity of low-birth-weight infants. Nowadays, surfactant therapy has become the standard therapy of newborn RDS and is deemed one of the most effective and safe medicines in the health system by the WHO.

\section{COMPONENTS AND PHYSIOLOGICAL EFFECTS}

Endogenous surfactant

Mammalian pulmonary surfactant has similarities in its chemical composition across various species, and hence can be used as a substitute in the human lung. Pulmonary surfactant is a mixture of $90 \%$ lipids and $10 \%$ proteins, which in its typical monolayer formation is essential for the function of gas exchange at the fluid -air interface of the internal lung surface.

With its hydrophobic and hydrophilic properties, it decreases surface tension, thus keeping the alveoli open during the expansion and contraction phases of the respiratory cycle. Surfactant is composed of $\sim 80-85 \%$ phospholipids, $5-10 \%$ neutral lipids and $8-10 \%$ protein, with $5-6 \%$ consisting of the four specific surfactant proteins. Eighty-five percent of the phospholipid fraction itself consists of phosphatidylcholines, the most important component $(40 \%)$ with the highest compaction properties being di-palmitoylphosphatidylcholine (DPPC); $11 \%$ consists of phosphatidylglyerol and phoshatidylinositol, which fluidize the lipid monolayer. The remaining fraction consists of various phospholipids with particular functions. Cholesterol is the major neutral lipid (85\%). The various lipid fractions are transported from the smooth endoplasmic reticulum into multivesicular bodies, they are assembled and stored with the other lipid and protein fractions in lamellar bodies, and then secreted into the alveolar space, forming tubular myelin and finally the dynamic surfactant monolayer at the fluid-air interface. Protein components of surfactant are apolipoproteins and a repertoire of specific proteins, called surfactant protein (SP)-A, $-B,-C$ and $-D$, which are produced by type II cells and contribute different essential surfactant functions.

The hydrophilic proteins (SPA, SPD), belonging to the collectin family, are multimeric macromolecular complexes and play a major role in the innate defense mechanisms by interacting with macrophages to enable phagocytosis of viruses and bacteria. In addition, SP-A enhances the activity of SP-B and SP-C and preserves the integrity of extensively packed lipid-protein complexes, thus optimizing surfactant performance under particularly demanding physiological conditions, and prevents

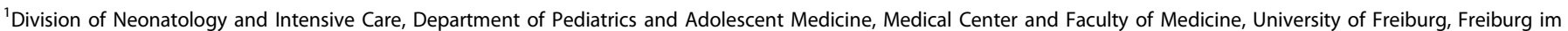

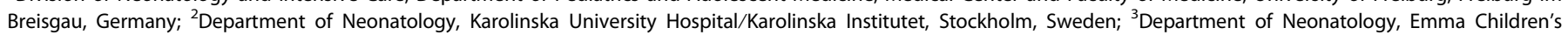

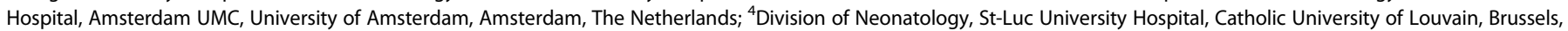
Belgium and ${ }^{5}$ Department of Pediatrics, University of California San Francisco, San Francisco, CA, USA

Correspondence: Roland Hentschel (roland.hentschel@uniklinik-freiburg.de)

Received: 24 June 2019 Revised: 15 December 2019 Accepted: 20 December 2019

Published online: 11 January 2020 
inhibition of surfactant by serum components. SP-D action on surfactant function is less clearly defined but could be important for promoting production of a surplus of surfactant in processes and situations where surfactant homeostasis is challenged. ${ }^{1}$ SP-B and SP-C are essential for spreading and for the physicochemical function of surfactant. SP-B is critical for adsorption of surfactant phospholipids into the fluid-air interface and the dynamic behavior of interfacial surfactant films subjected to the continuous compression-expansion cycling resulting from tidal respiration. ${ }^{1,2}$ Its critical role is well demonstrated by severe respiratory failure and neonatal death in case of genetically determined SP-B deficiency, either in human disease or in animal models. ${ }^{3}$ SP-C is able to promote adsorption and transfer of phospholipids into the fluid-air interface, and promotes the formation of multilamellar structures associated with the interface during compression of surfactant films close to functional residual capacity (FRC). ${ }^{4}$ Lack of $\mathrm{SP}-\mathrm{C}$ results in lung inflammation and pneumonia; however, it has only minor or delayed clinical effects in animal models, and SP-C mutation-associated lung disease in humans results from protein misfolding or mistrafficking rather than from haploinsufficiency. ${ }^{5}$ All proteins are also essential to keep the mixture of surfactant lipids and proteins in a liquid phase.

Critical tension-active properties have been shown to depend mainly on lipid composition, with over 50 different lipid molecules composing species-specific surfactant compounds with biophysical characteristics tailored not only to body temperature, but also to respiratory rate, and other biological and environmental parameters. ${ }^{2}$ Indeed, intracellular phospholipid transport disruption, induced by bi-allelic mutations of the $A B C A 3$ transporter, leads to early-onset, severe lung disease and often lethal respiratory failure in infancy. ${ }^{6}$ Conversely, specific proteins play a critical role in film formation and maintenance. The hydrophobic proteins modulate surfactant phase behavior and morphology independently from the lipid composition, but dependent on the coexistence of ordered and disordered phases.

While the biochemistry and molecular biology of surfactant has been the object of intensive laboratory research, showing how biochemical composition, molecular tri-dimensional structure, lipid-protein interactions and mechanical features of surfactant membranes and films correlate with surfactant biological function inside the lungs, most of these findings did not culminate in clinical research or practice applications so far.

Finally, complex mechanisms mediate alveolar surfactant catabolism and recycling by alveolar macrophages and type II pneumocytes. The granulocyte-macrophage-colony-stimulating factor (GM-CSF) pathway is central in these processes, as demonstrated by the development of pediatric-onset alveolar proteinosis in subjects with mutations of its receptor subunits CSF2RA and CSF2RB.?

Techniques for functional testing of surfactant

Since John Clements conducted the first direct measurements of pulmonary surfactant using his home-made Langmuir-Wilhelmy surface balance half a century ago, ${ }^{8}$ many more in vitro tensiometric techniques, such as the pulsating bubble surfactometry, captive bubble surfactometry, and the constrained sessile drop, have been developed to assess surfactant function. These techniques, providing a direct measure of surface tension properties, were complemented by biochemical or microscopic assays in amniotic fluid, gastric or tracheal aspirates: the lecithin/ sphingomyelin ratio, percentage of phosphatidylglycerol, the lamellar body count (LBC) and the stable microbubble test (SMT) gained a certain importance for prediction of RDS antenatally, ${ }^{9}$ need for intubation and surfactant early postnatally in preterm infants, or for diagnosis of secondary surfactant deficiency in infants and children. ${ }^{10,11}$

Since these laboratory assays were time-consuming, technically demanding, difficult to standardize and far from physiologic conditions they are rarely considered nowadays, also because they were validated only in small studies, but in particular since antenatal corticosteroids and postnatal surfactant administration both have dramatically altered the appearance of RDS in the past few centuries.

Even though it is still worth mentioning these techniques, not only for historic purpose, but also since the in vitro tensiometric techniques are relevant to studies on designing artificial surfactants, improving surfactant composition, or identifying factors, which may either have an impact on surfactant inhibition or on stability.

Recently, Ravasio et al. ${ }^{12}$ described a technique for measuring phospholipid (PL) surface film formation in multiwell plates containing fluorescence-labeled surfactant and a light quencher that allows high-throughput kinetic analyses at various concentrations of surfactant, and developed it into an assay, designated surfactant adsorption test (SAT). This innovative technique allows simultaneous analysis of multiple samples, making it suitable for clinical applications as well as for experimental testing of pharmacological compounds. Adapting this assay for clinical testing, Danhaive et al. ${ }^{13}$ demonstrated the correlation of surfactant film formation properties with SP-B content in preterm infants receiving late surfactant therapy for persisting respiratory failure. Using a similar technique, De Luca et al. ${ }^{14}$ described the positive effect of whole-body hypothermia on surfactant function after $48 \mathrm{~h}$ in term infants with hypoxic-ischemic encephalopathy and respiratory failure. Autilio et al. ${ }^{15}$ showed that SAT values could predict continuous positive airway pressure (CPAP) failure and the need for surfactant replacement in preterm infants $\leq 32$ weeks gestational age (GA) with RDS, compared to established clinical criteria and validated tests such as lung ultrasonography or LBC. This promising technique has the potentiality of being developed into a fast, standardized laboratory test, which would open the door to clinical applications.

Imaging techniques, immunochemistry, kinetics and homeostasis The spatial-temporal nature of ventilation within the lung as it aerates after birth is a key element in clinical management of RDS, and assessing the contribution of endogenous and exogenous surfactants in the process in vivo remains a major challenge. Various animal models have been developed for assessing regional surfactant distribution and pool sizes in vitro and in vivo with immunochemistry and chemical or radioactive isotope labeling. ${ }^{16}$ Phase contrast X-ray imaging allows visualizing and measuring the volume of air entering different regions of the lung on a breath-by-breath basis from birth on, thus assessing exogenous surfactant effect in real time. ${ }^{17}$ In part owing to danger of radiation damage, none of these techniques have been applied to in vivo dynamic measurements in humans so far.

Bronchoalveolar lavage has been a source for assessing surfactant composition by biochemical, immunochemical or cytology techniques. Quantitative measurements of SP-B and SP$C$ apoprotein fractions and mature peptides have been used as an index of alveolar maturation ${ }^{18}$ and as a screening tool for genetic surfactant homeostasis disorders and other pediatric interstitial lung diseases, with low SP-B level indicating SP-B deficiency, and low SP-C level suggesting mutations in SFTPC, NKX2.1 and $A B C A 3$ genes. $^{19}$

Surfactant kinetics has initially been studied by radioactive isotope or immunochemistry labeling in animal models. However, kinetics of surfactant phospholipid and protein synthesis and turnover can also be studied in vivo in infants by using the nonradioactive, stable isotopes ${ }^{13} \mathrm{C}$ and ${ }^{2} \mathrm{H}$ and analyzing serial tracheal aspirates with mass spectrometry. ${ }^{20}$ Term infants have a surfactant storage pool of approximately $100 \mathrm{mg} / \mathrm{kg}$ of surfactant, while preterm infants have an estimated pool size of $4-5 \mathrm{mg} / \mathrm{kg}$ at birth. Desaturated phosphatidylcholine fractional synthesis rate (FSR) and half-life were calculated to be 25 percent/day and $35 \mathrm{~h}$, 
respectively, in a cohort of newborns, but are highly variable, ranging from 16 to $177 \mathrm{~h}$ in published studies, depending on GA, postnatal age and other associated conditions. With the same method for exogenous surfactant PLs, an average half-life of $34 \mathrm{~h}$ and for SP-B an FSR and half-life of 30 percent/day and $21 \mathrm{~h}$, respectively, was determined. The stable isotope method was used to demonstrate disruption of endogenous surfactant homeostasis in RDS, congenital diaphragmatic hernia, meconium aspiration pneumonia, genetic surfactant dysfunction diseases and other pathologic conditions, ${ }^{21-24}$ but it can also assess the effects of exogenous surfactants in infants with RDS. ${ }^{25}$ Even though the stable isotope method is a powerful research tool and allows precise human in vivo measurements without biological toxicity it is too complex and time-consuming for routine applications.

\section{EXOGENOUS SURFACTANT REPLACEMENT—CLINICAL \\ EFFECTS}

Animal-derived surfactant preparations

Since the first studies of surfactant treatment for neonatal RDS in the 1960s, several surfactant preparations have been tested and compared in clinical trials. Currently, the only commercially available animal-derived surfactants are harvested either by lavage from bovine lungs (such as calfactant, bovactant, bovine lipid extract surfactant) or through mincing of porcine or bovine lung tissue, followed by centrifugation (such as poractant alfa and beractant).

Commercially available animal-derived surfactant preparations differ in composition and concentration of PLs and proteins. Poractant alfa uses liquid chromatography to extract only polar lipids and contains the highest total concentrations of phospholipids and SP-B. This in turn means that the volume necessary to administer the same amount of phospholipids intratracheally is lowest for poractant alfa and allows for administration of a higher dose, up to $200 \mathrm{mg} / \mathrm{kg}$, even in the smallest infants.

A large retrospective study comparing more than 14,000 infants treated with poractant alfa, beractant or calfactant reported a significantly lower overall mortality for infants with birth weights $500-749 \mathrm{~g}$ when treated with poractant alfa, ${ }^{26}$ but this difference was not found in another large retrospective analysis of a more mature cohort with $>50 \%$ of infants $>31$ weeks GA. ${ }^{27}$

The size of the first dose of surfactant may be more important for clinical response than the source of surfactant. Infants treated with beractant were more often nonresponders if given a lower dose of $50 \mathrm{mg} / \mathrm{kg}$, compared to a higher dose of $100 \mathrm{mg} / \mathrm{kg}^{28}$ Doses higher than $100 \mathrm{mg} / \mathrm{kg}$ of beractant are difficult to administer due to the comparatively low concentration of surfactant in the solution. For poractant alfa a high dose of 200 $\mathrm{mg} / \mathrm{kg}$, as compared to a lower dose of $100 \mathrm{mg} / \mathrm{kg}$, led to better survival and decreased risk for complications such as persistent ductus arteriosus (PDA) and intraventricular hemorrhage. ${ }^{29} \mathrm{~A}$ higher first dose also reduces the need for repeat doses. Older studies, before implementation of antenatal steroids and with other ventilation strategies, suggested that multiple doses of surfactant had additive effects in reducing complications such as pneumothorax. Recent guidelines do not recommend repeat doses routinely, but advise that a second (and third) dose should be given to infants with ongoing or progressing respiratory distress. Surfactant treatment during CPAP, such as INSURE (INtubation-SURfactant-Extubation) and LISA (Less Invasive Surfactant Administration), may reduce the need for repeat doses. ${ }^{30}$

A large recent meta-analysis, including 1483 neonates in 14 trials, found that $200 \mathrm{mg} / \mathrm{kg}$ poractant alfa was associated with lower frequency of retreatment, but also with lower rate of air leaks and lung hemorrhage, bronchopulmonary dysplasia (BPD) and lower mortality, when compared to bovine surfactants at the recommended lower respective doses. ${ }^{31}$ An impact on retreatment and BPD was most pronounced in the lowest and highest GA groups. A meta-analysis by Singh et al. ${ }^{29}$ had given comparable evidence. These results might suggest that the higher dose or concentration of phospholipids in poractant alfa may be important for optimal efficacy; however, there is a lack of doseequivalent comparison groups to reliably answer this question, and there is the general flaw of meta-analyses that differences in indication and pattern of use at different centers may weaken the validity of results. Hence, it is not clear whether differing results are due to chemical composition, resistance to inactivation, or source of surfactant.

\section{Synthetic surfactant preparations}

Recognizing the production limitations, costs and biological risks of animal-derived surfactants, many attempts have been made to produce synthetic surfactants for replacement therapy. The characteristics of an optimal surfactant include reduction of surface tension to values of $\sim 1-2 \mathrm{mN} / \mathrm{m}$ under compression, rapid adsorption and film formation at the alveolar gas-liquid interface, maintenance of tension-active properties over several repeated compression-expansion cycles during breathing, and maintenance of its properties at lower concentrations and in the presence of inhibitors.

A fully synthetic surfactant is appealing, because it eliminates concerns about the animal origin of mammalian surfactants, such as the potential risk of immunological or infectious side effects, and it also secures more predictable and consistent concentrations of all components in the surfactant preparation.

First-generation synthetic surfactants, that consisted of lipidonly, protein-free mixtures, were of limited success and inferior to animal-derived surfactants ${ }^{32}$ in treating RDS because of their inability to adsorb at the alveolar fluid-air interface. This latter drawback was overcome with colfosceryl palmitate (Exosurf ${ }^{\circledR}$, approved worldwide and commercially available 1991-2003), which besides DPPC to lower surface tension also contained hexadecanol to promote adsorption and tyloxapol to facilitate dispersion. This second-generation synthetic surfactant was efficacious in treating RDS in preterm infants, ${ }^{33}$ but proved to be inferior to animal-derived surfactants in randomized control trials, and was finally withdrawn from the market.

Given the inferiority of protein-free synthetic surfactants, two third-generation synthetic surfactants (lusupultide and lucinactant) have been developed by adding functionally important proteins.

Lusupultide (Venticute ${ }^{\circledR}$ ), which contains recombinant SP-C, has only been tried in adults with acute respiratory distress syndrome (ARDS), but showed disappointing results despite some benefits in oxygenation and survival in patients with pneumonia or aspiration, and was never commercialized.

Lucinactant incorporated sinapultide (KL4 acetate), a peptide analog mimicking SP-B, in a phospholipid mixture closer to human surfactant composition. This compound showed in vitro functional characteristics equal or superior to endogenous or animal-derived surfactants, and was not inferior in vivo; thus, it was approved and commercialized as Surfaxin ${ }^{\circledR} .34,35$

Lucinactant has been evaluated against beractant and poractant alfa in two randomized trials and, although the second trial was stopped prematurely, the results suggest that it was safe, and outcome was comparable to animal-derived surfactants. ${ }^{34,35}$ Even though some meta-analyses showed that lucinactant treatment resulted in higher survival than other commercial products, possibly due to a better resistance to inactivation and degradation, it failed to reach commercial success. The drawbacks of the lucinactant preparation was its high viscosity at room temperature and a gel formulation, which required heating, mixing and subsequent cooling to body temperature before administration. Also, the dose-equivalent volume was approximately 2.5 times that of poractant alfa. Surfaxin was withdrawn from the European 
market in 2006, and production was completely stopped by the US manufacturer in 2015.

Lucinactant is currently under development as an aerosolized surfactant (Aerosurf ${ }^{\circledR}$ ), having reached preclinical testing. ${ }^{36}$

Recently, CHF5633, a third-generation compound combining a $0.2 \%$ SP-B analog and a $1.5 \%$ SP-C analog in a $1: 1$ DPPC: palmitoyloleoylphosphatidylglycerol mixture, yielded promising results for RDS therapy in a phase- 1 human trial, ${ }^{37}$ and is currently undergoing a multicenter RCT versus animal-derived poractant alfa. In vivo stable isotope animal studies showed a considerable delay in catabolism and enhanced phospholipid recycling of CHF5633 compared to poractant alfa. ${ }^{38}$ Also, in vitro studies showed decreased proinflammatory cytokine synthesis in macrophages in response to CHF5633, ${ }^{39}$ suggesting the possibility of better efficacy in preventing chronic lung disease in preterm infants with RDS and broader indications for surfactant replacement therapy, such as neonatal or pediatric ARDS. ${ }^{40}$

Lambs treated with CHF5633 for RDS have better lung and brain injury scores than those treated with poractant alfa. The first phase-I human trial in 40 infants with 27-34 weeks GA reports rapid and sustained improvement in oxygen requirement for $98 \%$ of the infants, good tolerability and no unexpected adverse events. ${ }^{37}$ A phase-Il multicenter double-blinded clinical trial comparing CHF5633 with poractant alfa is ongoing (ClinicalTrials.gov NCT02452476).

\section{Additives for surfactants}

Surfactant consists of the two major subfractions called large aggregates (LA) and small aggregates (SA). LA surfactant is able to lower alveolar surface tension, but SA surfactant is not surface active and is the metabolic product of the LA fraction. ${ }^{41}$ Factors enhancing the conversion of LA to SA will therefore compromise surfactant function.

Anionic polymers such as dextran and hyaluronan have the property of enhancing and restoring interface adsorption of surfactant LA. Endogenous and animal-derived surfactants supplemented with hyaluronan showed enhanced resistance to inactivation by meconium, cholesterol or serum, offering the potential for clinical improvements in secondary surfactant deficiencies due to ARDS, pulmonary hemorrhage or aspiration. ${ }^{42}$

Due to its adsorption and spreading characteristics, surfactant is a potential vehicle for airway-targeted medications. ${ }^{43}$ Budesonide, a synthetic glucocorticoid, which undergoes a $90 \%$ first-pass hepatic catabolism, is widely used as a nebulized drug for asthma. It was used as an alternative to systemic steroids in the prevention and treatment of BPD, but no benefit could be demonstrated for the latter. ${ }^{44,45}$ Addition of budesonide to surfactant does not alter its functional properties and showed beneficial anti-inflammatory effects in animal models. ${ }^{46}$ In two successive RCTs, Yeh et al. tested a budesonide-surfactant mixture for prevention of BPD in preterm infants with RDS, showing a striking reduction of BPD with no long-term neurological side effects. ${ }^{47,48}$

However, the unusually high incidence of BPD in their study population may limit the value of their conclusion. Also, these results have not been reproduced so far by other groups; however, a larger, US-based RCT of budesonide-supplemented surfactant for prevention of BPD is currently ongoing (SASSIE trial: ClinicalTrials.gov NCT02907593).

\section{CLINICAL PRACTICE}

New modes of administration

Different modes of surfactant administration have been studied with regard to impact on mechanical properties of the lung and gas exchange. The standard approach to administer surfactant is instillation via the endotracheal tube (ETT) in the mechanically ventilated infant with RDS. This allows rapid surfactant bolus application, resulting usually in a more homogenous surfactant distribution, compared to slow infusion of surfactant, as evidenced from animal studies. ${ }^{49-51}$ However, whether a plug forms coincidentally before the next inspiration, which is a prerequisite for a good surfactant distribution after bolus administration, ${ }^{50}$ cannot be anticipated. Surfactant obviously needs a few minutes to dissipate into the typical monolayer along bronchial and alveolar surfaces, which is essential for its physicochemical properties. However, an immediate change of resistance and the risk of disturbed blood pressure and heart rate, as well as bronchus obstruction, remain unsolved problems ${ }^{49,52-54}$; so, in individual cases slow surfactant infusion might be preferable. ${ }^{49}$ Against this background inhalation techniques continue to attract interest, in particular because intubation is not required.

Since mechanical ventilation is associated with barotrauma and increased risk for ventilator-associated infections, noninvasive modes to administer surfactant were sought to minimize need for endotracheal intubation or duration of mechanical ventilation. ${ }^{55,56}$

The INSURE procedure comprises intubation followed by surfactant administration and early extubation. This approach uses short acting sedatives for intubation allowing extubation to noninvasive respiratory support right after surfactant administration. Since the studies by Verder et al. ${ }^{55}$ this approach has been widely adopted, by which the period of mechanical ventilation is minimized. Reversal of sedation or use of very short acting sedatives allow for rapid return to spontaneous breathing. ${ }^{57}$ However, only about $30 \%$ of preterm infants below 32 weeks GA are successfully treated with the INSURE procedure, whereas two out of three patients require longer periods of ventilation or reintubation, due to effect of sedatives, poor gas exchange despite continuous mechanical ventilation during the procedure, or exhaustion. ${ }^{58,59}$

More recently LISA was introduced, and this technique was adopted quickly into clinical practice. ${ }^{56,60}$ In this modification surfactant is delivered through a thin, flexible feeding tube or a catheter placed into the trachea during spontaneous breathing, often supported with CPAP, eliminating the need to intubate with an ETT. Usually little or no sedation is given for the procedure, which has been described as a rapid injection technique, but also as a slow infusion over $1-3$ min. $^{61}$ Depending on the risk of surfactant reflux due to the leak at the level of the larynx, a very slow application of surfactant may be preferred, and nonhomogenous distribution does not seem to be a problem with this technique. ${ }^{61}$ Also, coughing of the infant during the procedure may cause reflux into the pharynx and hamper deposition of the whole amount of surfactant in the lung.

Both newer modes of surfactant therapy have proven to reduce the duration of invasive mechanical ventilation. Several randomized trials showed an effectivity at least equivalent to the classical approach with intubation and mechanical ventilation. ${ }^{62,63}$ Inclusion of larger numbers of infants through meta-analyses suggests a reduction in BPD and mortality with the new modalities of surfactant administration. ${ }^{63}$ Therefore, combination of surfactant therapy and noninvasive respiratory support may be a valuable approach to further improve long-term outcomes.

Subgroup analysis of the NINSAPP trial, which compared invasive surfactant administration with the LISA technique, suggested that very immature infants may benefit less from the noninvasive approach compared to the subgroup of $>24$ completed weeks of $\mathrm{GA}^{62}$ Also, the severity of RDS might influence the choice, by which mode surfactant is given. Further studies should try to unravel how to proceed in case of LISA or INSURE failure, defined for instance by persistent high oxygen demands.

Surfactant has been given into the pharynx before the first breath, $^{64}$ via laryngeal mask $^{65}$ or by nebulization ${ }^{66}$ either experimentally or in small trials. Deposition of a satisfactory dose is the main challenge hampering these approaches. However, with 
new technical developments, these techniques may play a role in the future.

Factors improving or impeding effect of endogenous or exogenous surfactant

Consistent with animal data human studies have clearly shown that antenatal corticosteroid (ANS) treatment has significant beneficial effects on the outcome of preterm infants, reducing incidence and severity of RDS, but also mortality as one of the most important effects. ${ }^{67}$ This reduced mortality is in part mediated by an improved lung function at the time of birth. Animal models have shown that ANS accelerate lung maturation by thinning of the walls between the alveolar and vascular compartment and by speeding up maturation of the surfactant producing type II pneumocytes. ${ }^{68}$ As a result lung volumes and mechanics are improved. Furthermore, ANS treatment enhances the effect of exogenous surfactant on these outcomes and on lung function. ${ }^{69}$ In summary, ANS together with surfactant independently, but also additively, reduce mortality, severity of RDS, and air leak of preterm infants. Mortality is reduced, but there is no effect on neurodevelopmental outcome, and rate of BPD is reduced only in single studies.

The most important effect of exogenous surfactant is lowering of the alveolar surface tension thereby improving lung volume, lung mechanics and gas exchange. However, the effect of exogenous surfactant on these outcomes may differ between patients, and several factors impacting the response to exogenous surfactant have been identified.

The timing of surfactant treatment after birth can also impact its efficacy. Several meta-analyses have shown that delaying surfactant treatment after birth will have a negative impact on its efficacy. ${ }^{70,71}$ It has been suggested that this difference in favor of early treatment may be explained by a more optimal distribution of surfactant in a still fluid-filled lung, and the lack of ventilatorinduced lung injury accompanied by fluid and protein influx into the alveolar space. However, it is important to mention that these negative effects of delayed surfactant treatment have mainly been observed in trials using invasive mechanical ventilation as initial strategy for respiratory support after birth. Nowadays, many centers have adopted noninvasive ventilation as the primary mode postnatally, and studies have shown that delaying surfactant treatment under these circumstances does not have a negative effect on its efficacy, when compared with primary invasive respiratory support combined with early (prophylactic) surfactant treatment. ${ }^{70}$

Surfactant function can be impeded by several pathophysiologic conditions.

Secondary surfactant deficiency from surfactant inactivation may occur with aspiration syndrome, pulmonary hemorrhage, pneumonia or ARDS.

An aspiration syndrome may be due to ingestion of meconium, ${ }^{72}$ blood $^{73}$ milk $^{74}$ or bile ${ }^{75}$ into the lung. Part of the deleterious effects of meconium aspiration syndrome is exerted by inactivation of alveolar surfactant and activation of severe inflammation causing pneumonitis. ${ }^{72}$

Blood components like hemoglobin, entering the lung either with sangineous amnion fluid or following pulmonary hemorrhage, rapidly inactivate surfactant causing secondary surfactant deficiency and severe decline in lung function. ${ }^{76}$ Lung lavage with diluted surfactant and subsequent refilling of the surfactant pool with an adequate dose of animal-derived surfactant can be performed to overcome serious respiratory compromise exerted

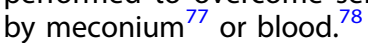

Disturbance of surfactant homeostasis in the presence of chorioamnionitis or pneumonia results from inactivation of surfactant with leakage of plasma proteins into the airspaces and influx of inflammatory cells causing cytokine release and inflammation. ${ }^{79}$ A similar effect is part of the pathophysiologic cascade in ARDS. Surfactant is frequently needed in inflammation processes, like chorioamnionitis, pneumonia or ARDS. However, while the role of surfactant therapy is well established to improve gas exchange and reduce mortality in primary surfactant deficiency in the preterm infant, its role in surfactant inactivation through inflammation is less clear: response is more unpredictable and often slower; also repeated doses of surfactant may be needed. ${ }^{80}$

\section{Assisted ventilation after surfactant administration}

Mechanical ventilation of preterm infants is associated with volutrauma and hyperoxia, leading to lung damage. This triggers an inflammation process, which activates cellular response and release of cytokines and proteases, harbingers of BPD. This sequence of injury can be attenuated or even abrogated by surfactant administration.

Animal data suggest that large tidal volumes $(\mathrm{Vt})$ delivered shortly after birth during respiratory support of preterm lambs have a negative impact on surfactant response. ${ }^{81}$ However, there are no data to support this in humans.

Animal studies have also shown that injurious invasive mechanical ventilation increases conversion of SA to LA surfactant, which leads to leakage of proteins into the alveolar space and reduces surfactant function. Applying so-called lung-protective ventilation strategies can attenuate this process. ${ }^{82}$ This observation may in part explain why several trials comparing highfrequency ventilation (HFV) to conventional mechanical ventilation (CMV) have shown that infants treated with (lung protective) HFV require less repeat treatments with surfactant, which may indirectly indicate a better preservation of the first surfactant dose. ${ }^{83}$

Lack of surfactant, due to premature birth, hampers constant lung expansion, so continuous distending pressure from intermittent mandatory ventilation (IMV) or CPAP and administration of exogenous surfactant is required.

A homogenous distribution across the whole compartment of terminal bronchioli and alveoli would be optimal; however, surfactant will not reach areas, which are filled with debris or are collapsed, so a certain distending pressure is required immediately before and after surfactant administration.

Unless surfactant is given via a side port of the ETT during continuous ventilation or CPAP, surfactant administration usually requires disconnection of the patient from the ventilator for a short period of time, during which alveoli will collapse. So a static distending pressure via ventilation bag to re-open the lung and facilitate distribution of surfactant into the periphery after disconnection may be advantageous.

Immediate increase in oxygenation, following surfactant administration, is probably the result of increased FRC, not of altered lung mechanics, ${ }^{84}$ so $\mathrm{FiO}_{2}$ is always the first parameter of ventilation, which can be reduced.

The subsequent ventilation most often requires increased peak inspiratory pressure (PIP) to yield at least minimum ventilation in the first minutes after surfactant treatment ${ }^{53,54}$ or to overcome the frequent phenomenon of total bronchial obstruction. ${ }^{54}$ This period of increased resistance requires a long inspiratory time, relatively long expiratory time, and thus a low frequency, which contrasts to findings from a model of excised rat lungs showing that higher frequencies in the range of $60 / \mathrm{min}$ improved homogenous distribution of surfactant to different lung lobes. ${ }^{85}$ In clinical practice change of $\mathrm{pO}_{2}$ and $\mathrm{pCO}_{2}$ will most often require an immediate intervention by intensification of ventilation.

Positive end-expiratory pressure (PEEP) must be high enough to keep alveoli open during expiration, and from the clinical perspective PIP must be high enough to yield visible chest expansions after surfactant administration.

To some extent ventilation modes with volume guarantee may help to overcome the problem of rapidly changing lung 
mechanics after surfactant, resulting in changing Vt, but PIP must be set high enough. ${ }^{54}$

Within a timeframe of about $30-60 \mathrm{~min}$, compliance will increase and resistance will return to baseline. ${ }^{53}$ It is mandatory to notice this development by either watching thorax expansion, which can change rapidly, or to notice increasing $\mathrm{Vt}$, if measured. A great and timely reduction of PIP and PEEP is then mandatory to avoid alveolar overdistension and extra-alveolar leakage. At this time point frequency can eventually be increased.

Surfactant administration while using HFV with the "open lungconcept" has also shown promising results to keep the lung open in surfactant deficiency; continuous distending pressure could be reduced, ${ }^{86}$ comparable to the effect during CMV, where FRC increases immediately and compliance increases after a short time. ${ }^{53}$

For effective and lung-protective ventilation in these most vulnerable preterm infants, the time-resolved mechanics of the inspiratory and expiratory part of the ventilation cycle should be taken into consideration. In this respect total bronchial and bronchiolar resistance and local bronchiolar and alveolar compliance are the most important determinants.

Hemodynamic changes and extrapulmonary effects

In animal and human studies surfactant administration leads to immediate changes in hemodynamics. It is speculated that surfactant might have a "pharmacological" effect causing vasodilation. In a dose-dependent manner mean arterial blood pressure decreases after surfactant, due to systemic vasodilation, but can be partly compensated by an increase in cardiac output. On the other hand, these effects are difficult to distinguish from effects resulting from the frequently observed increase in $\mathrm{pCO}_{2}$, leading in particular to cerebral vasodilation and a decrease of left-toright-shunt across the PDA after increase of pulmonary vascular resistance. But there are also conflicting results regarding change of cerebral blood flow velocity and/or regional cerebral oxygenation immediately after surfactant and uncertainty about pulmonary arterial pressure and pulmonary blood flow. These parameters can be measured only indirectly, but differences in timing of measurement, surfactant dose and persistence of PDA may also lead to varying results. Alarming effects on hemodynamics at about 20-30 min after surfactant administration often are the result of a compromised venous return due to an overinflated lung, if PIP and PEEP are not reduced adequately.

\section{SUMMARY}

Over 50 years of basic research on surfactant biology and homeostasis have yielded one of the greatest breakthroughs in neonatology, surfactant replacement therapy, which has led to a spectacular increase in survival, pushing back the boundaries of premature viability, and improving short- and long-term morbidity of preterm infants. However, translation of basic research into clinical applications remains often challenging, due to the complexity and extreme sensitivity of the surfactant system, the lack of clinically applicable assays and techniques, and the fragility of the premature infant. Translational research efforts, which aim at closing this gap, hold the potential for future advances, which may go well beyond prematurity and the neonatal period.

\section{AUTHOR CONTRIBUTIONS}

R.H. framed the review concept, drafted part of the article, compiled all parts of the manuscript, and revised it critically for important intellectual content. K.B. drafted part of the article. A.v.K. drafted part of the article and helped revise the manuscript critically for important intellectual content. H.F. drafted part of the article. O.D. drafted part of the article and revised the manuscript critically for important intellectual content. All authors gave their final approval for this paper for publication.

\section{ADDITIONAL INFORMATION}

Competing interests The authors declare no competing interests.

Publisher's note Springer Nature remains neutral with regard to jurisdictional claims in published maps and institutional affiliations.

\section{REFERENCES}

1. Parra, E. \& Perez-Gil, J. Composition, structure and mechanical properties define performance of pulmonary surfactant membranes and films. Chem. Phys. Lipids 185, 153-175 (2015)

2. Suri, L. N. et al. Adaptation to low body temperature influences pulmonary surfactant composition thereby increasing fluidity while maintaining appropriately ordered membrane structure and surface activity. Biochim. Biophys. Acta 1818, 1581-1589 (2012).

3. Nogee, L. M. et al. A mutation in the surfactant protein B gene responsible for fatal neonatal respiratory disease in multiple kindreds. J. Clin. Invest. 93 1860-1863 (1994).

4. Parra, E. et al. A combined action of pulmonary surfactant proteins SP-B and SP-C modulates permeability and dynamics of phospholipid membranes. Biochem. J. 438, 555-564 (2011).

5. Peca, D. et al. Clinical and ultrastructural spectrum of diffuse lung disease associated with surfactant protein C mutations. Eur. J. Hum. Genet. 23, 1033-1041 (2015).

6. Peca, D. et al. $A B C A 3$, a key player in neonatal respiratory transition and genetic disorders of the surfactant system. Biochem. Soc. Trans. 43, 913-919 (2015).

7. McCarthy, C. et al. Statin as a novel pharmacotherapy of pulmonary alveolar proteinosis. Nat. Commun. 9, 3127 (2018).

8. Clements, J. A. Surface phenomena in relation to pulmonary function. Physiologist 5, 11-28 (1962).

9. Stichtenoth, G. et al. Surface tension of airway aspirates withdrawn during neonatal resuscitation reflects lung maturity. Pediatr. Pulmonol. 49, 751-756 (2014).

10. Bhatia, R. et al. The stable microbubble test for determining continuous positive airway pressure (CPAP) success in very preterm infants receiving nasal CPAP from birth. Neonatology 104, 188-193 (2013).

11. Vieira, A. C. et al. Lamellar body count and stable microbubble test on tracheal aspirates from infants for the diagnosis of respiratory distress syndrome. Pediatr. Crit. Care Med. 13, 178-182 (2012).

12. Ravasio, A., Cruz, A., Perez-Gil, J. \& Haller, T. High-throughput evaluation of pulmonary surfactant adsorption and surface film formation. J. Lipid Res. 49, 2479-2488 (2008).

13. Danhaive, O. et al. Surface film formation in vitro by infant and therapeutic surfactants: role of surfactant protein B. Pediatr. Res. 77, 340-346 (2015).

14. De Luca, D. et al. Hypothermia and meconium aspiration syndrome: International Multicenter Retrospective Cohort Study. Am. J. Respir. Crit. Care Med. 194 381-384 (2016).

15. Autilio, C. et al. A noninvasive surfactant adsorption test predicting the need for surfactant therapy in preterm infants treated with continuous positive airway pressure. J. Pediatr. 182, 66-73 (2017).

16. Hutten, M. C. et al. Nebulization of Poractant alfa via a vibrating membrane nebulizer in spontaneously breathing preterm lambs with binasal continuous positive pressure ventilation. Pediatr. Res. 78, 664-669 (2015).

17. Siew, M. L. et al. Surfactant increases the uniformity of lung aeration at birth in ventilated preterm rabbits. Pediatr. Res. 70, 50-55 (2011).

18. Hamvas, A. et al. Developmental and genetic regulation of human surfactant protein B in vivo. Neonatology 95, 117-124 (2009).

19. Griese, M. et al. Surfactant proteins in pediatric interstitial lung disease. Pediatr. Res. 79, 34-41 (2016).

20. Cogo, P. E. et al. Endogenous surfactant metabolism in critically ill infants measured with stable isotope labeled fatty acids. Pediatr. Res. 45, 242-246 (1999).

21. Torresin, M. et al. Exogenous surfactant kinetics in infant respiratory distress syndrome: a novel method with stable isotopes. Am. J. Respir. Crit. Care Med. 161, 584-589 (2000).

22. Cogo, P. et al. Surfactant-associated protein B kinetics in vivo in newborn infants by stable isotopes. Pediatr. Res. 57, 519-522 (2005).

23. Cogo, P. E. et al. Impaired surfactant protein B synthesis in infants with congenital diaphragmatic hernia. Eur. Respir. J. 41, 677-682 (2013).

24. D'Aronco, S. et al. Surfactant protein B and A concentrations are increased in neonatal pneumonia. Pediatr. Res. 78, 401-406 (2015).

25. Cogo, P. E. et al. Dosing of porcine surfactant: effect on kinetics and gas exchange in respiratory distress syndrome. Pediatrics 124, e950-e957 (2009).

26. Ramanathan, R., Bhatia, J. J., Sekar, K. \& Ernst, F. R. Mortality in preterm infants with respiratory distress syndrome treated with poractant alfa, calfactant or beractant: a retrospective study. J. Perinatol. 33, 119-125 (2013). 
27. Trembath, A. et al. Comparative effectiveness of surfactant preparations in premature infants. J. Pediatr. 163, 955-960 (2013).

28. Gortner, L. et al. High-dose versus low-dose bovine surfactant treatment in very premature infants. Acta Paediatr. 83, 135-141 (1994).

29. Singh, N. et al. Comparison of animal-derived surfactants for the prevention and treatment of respiratory distress syndrome in preterm infants. Cochrane Database Syst. Rev. 12, CD010249 (2015).

30. Bohlin, K. et al. Implementation of surfactant treatment during continuous positive airway pressure. J. Perinatol. 27, 422-427 (2007).

31. Tridente, A., De Martino, L. \& De Luca, D. Porcine vs bovine surfactant therapy forpreterm neonates with RDS: systematic review with biological plausibility andpragmatic meta-analysis of respiratory outcomes. Respir. Res. [online]. https:// doi.org/10.1186/s12931-019-0979-0.

32. Ardell, S., Pfister, R. H. \& Soll, R. Animal derived surfactant extract versus protein free synthetic surfactant for the prevention and treatment of respiratory distress syndrome. Cochrane Database Syst. Rev. 8, CD000144 (2015).

33. Phibbs, R. H. et al. Initial clinical trial of EXOSURF, a protein-free synthetic surfactant, for the prophylaxis and early treatment of hyaline membrane disease. Pediatrics 88, 1-9 (1991).

34. Sinha, S. K. et al. A multicenter, randomized, controlled trial of lucinactant versus poractant alfa among very premature infants at high risk for respiratory distress syndrome. Pediatrics 115, 1030-1038 (2005).

35. Moya, F. R. et al. A multicenter, randomized, masked, comparison trial of lucinactant, colfosceril palmitate, and beractant for the prevention of respiratory distress syndrome among very preterm infants. Pediatrics 115, 1018-1029 (2005).

36. Finer, N. N. et al. An open label, pilot study of Aerosurf ${ }^{\circledR}$ combined with nCPAP to prevent RDS in preterm neonates. Aerosol Med. Pulm. Drug Deliv. 23, 303-309 (2010).

37. Sweet, D. G. et al. A first-in-human clinical study of a new SP-B and SP-C enriched synthetic surfactant (CHF5633) in preterm babies with respiratory distress syndrome. Arch. Dis. Child Fetal Neonatal Ed. 102, F497-F503 (2017).

38. Madsen, J. et al. Metabolism of a synthetic compared with a natural therapeutic pulmonary surfactant in adult mice. J. Lipid Res. 59, 1880-1892 (2018).

39. Glaser, K. et al. Anti-inflammatory effects of the new generation synthetic surfactant CHF5633 on Ureaplasma-induced cytokine responses in human monocytes. Expert Rev. Anti. Infect. Ther. 15, 181-189 (2017).

40. De Luca, D. et al. The Montreux definition of neonatal ARDS: biological and clinical background behind the description of a new entity. Lancet Respir. Med. 5, 657-666 (2017)

41. Yamada, T., Ikegami, M. \& Jobe, A. H. Effects of surfactant subfractions on preterm rabbit lung function. Pediatr. Res. 27, 592-598 (1990).

42. Taeusch, H. W., Dybbro, E. \& Lu, K. W. Pulmonary surfactant adsorption is increased by hyaluronan or polyethylene glycol. Colloids Surf. B Biointerfaces $\mathbf{6 2}$, 243-249 (2008).

43. Fajardo, C. et al. Surfactant versus saline as a vehicle for corticosteroid delivery to the lungs of ventilated rabbits. Pediatr. Res. 43, 542-547 (1998).

44. Shah, S. S., Ohlsson, A., Halliday, H. L. \& Shah, V. S. Inhaled versus systemic corticosteroids for the treatment of bronchopulmonary dysplasia in ventilated very low birth weight preterm infants. Cochrane Database Syst. Rev. 10, CD002057 (2017).

45. Onland, W., Offringa, M. \& van Kaam, A. Late ( $>/=7$ days) inhalation corticosteroids to reduce bronchopulmonary dysplasia in preterm infants. Cochrane Database Syst. Rev. 8, CD002311 (2017).

46. Kothe, T. B. et al. Effects of budesonide and surfactant in preterm fetal sheep. Am. J. Physiol. Lung Cell Mol. Physiol. 315, L193-L201 (2018).

47. Yeh, T. F. et al. Early intratracheal instillation of budesonide using surfactant as a vehicle to prevent chronic lung disease in preterm infants: a pilot study. Pediatrics 121, e1310-e1318 (2008).

48. Yeh, T. F. et al. Intratracheal administration of budesonide/surfactant to prevent bronchopulmonary dysplasia. Am. J. Respir. Crit. Care Med. 193, 86-95 (2016).

49. Fernandez-Ruanova, M. B. et al. Comparison of rapid bolus instillation with simplified slow administration of surfactant in lung lavaged rats. Pediatr. Pulmonol. 26, 129-134 (1998).

50. Cassidy, K. J. et al. A rat lung model of instilled liquid transport in the pulmonary airways. J. Appl. Physiol. (1985) 90, 1955-1967 (2001).

51. Ueda, T., Ikegami, M., Rider, E. D. \& Jobe, A. H. Distribution of surfactant and ventilation in surfactant-treated preterm lambs. J. Appl. Physiol. (1985) 76, 45-55 (1994).

52. Alvarez, F. J. et al. The effects of multiple small doses of exogenous surfactant on experimental respiratory failure induced by lung lavage in rats. Acta Anaesthesiol. Scand. 39, 970-974 (1995).

53. Hentschel, R. et al. Sequential changes in compliance and resistance after bolus administration or slow infusion of surfactant in preterm infants. Intensive Care Med. 28, 622-628 (2002)
54. Wheeler, K. I., Davis, P. G., Kamlin, C. O. \& Morley, C. J. Assist control volume guarantee ventilation during surfactant administration. Arch. Dis. Child Fetal Neonatal Ed. 94, F336-F338 (2009).

55. Verder, $\mathrm{H}$. et al. Nasal continuous positive airway pressure and early surfactant therapy for respiratory distress syndrome in newborns of less than 30 weeks' gestation. Pediatrics 103, E24 (1999).

56. Kribs, A. et al. Early administration of surfactant in spontaneous breathing with nCPAP: feasibility and outcome in extremely premature infants (postmenstrual age $</=27$ weeks). Paediatr. Anaesth. 17, 364-369 (2007).

57. Elmekkawi, A., Abdelgadir, D., Van, D. J., Choudhury, J. \& Dunn, M. Use of naloxone to minimize extubation failure after premedication for INSURE procedure in preterm neonates. J. Neonatal Perinat. Med. 9, 363-370 (2016).

58. Brix, N, et al. Predictors for an unsuccessful INtubation-SURfactant-Extubation procedure: a cohort study. BMC Pediatr [online]. https://doi.org/10.1186/14712431-14-155 (2014).

59. Dani, C., Corsini, I. \& Poggi, C. Risk factors for intubation-surfactant-extubation (INSURE) failure and multiple INSURE strategy in preterm infants. Early Hum. Dev. 88(Suppl 1), 3-4 (2012).

60. Klotz, D., Porcaro, U., Fleck, T. \& Fuchs, H. European perspective on less invasive surfactant administration-a survey. Eur. J. Pediatr. 176, 147-154 (2017).

61. van der Burg, P. S. et al. Effect of minimally invasive surfactant therapy on lung volume and ventilation in preterm infants. J. Pediatr. 170, 67-72 (2016).

62. Kribs, A. et al. Nonintubated surfactant application vs conventional therapy in extremely preterm infants: a randomized clinical trial. JAMA Pediatr. 169, 723-730 (2015).

63. Ali, E. et al. New modalities to deliver surfactant in premature infants: a systematic review and meta-analysis. J. Matern. Fetal Neonatal Med. 29, 3519-3524 (2016).

64. Lamberska, T. et al. Oropharyngeal surfactant can improve initial stabilisation and reduce rescue intubation in infants born below 25 weeks of gestation. Acta Paediatr. 107, 73-78 (2018).

65. Pinheiro, J. M., Santana-Rivas, Q. \& Pezzano, C. Randomized trial of laryngeal mask airway versus endotracheal intubation for surfactant delivery. J. Perinatol. 36, 196-201 (2016)

66. Minocchieri, S., Berry, C. A. \& Pillow, J. J. Nebulised surfactant to reduce severity of respiratory distress: a blinded, parallel, randomised controlled trial. Arch. Dis. Child Fetal Neonatal Ed. 104, F313-F319 (2018).

67. Vyas, J. \& Kotecha, S. Effects of antenatal and postnatal corticosteroids on the preterm lung. Arch. Dis. Child 77, F147-F150 (1997).

68. Gladstone, I. M., Mercurio, M. R., Devenny, S. G. \& Jacobs, H. C. Antenatal steroids, postnatal surfactant, and pulmonary function in premature rabbits. J. Appl. Physiol. 67, 1377-1382 (1989).

69. Farrell, E. E. et al. Impact of antenatal dexamethasone administration on respiratory distress syndrome in surfactant-treated infants. Am. J. Obstet. Gynecol. 161, 628-633 (1989)

70. Rojas-Reyes, M. X., Morley, C. J. \& Soll, R. Prophylactic versus selective use of surfactant in preventing morbidity and mortality in preterm infants. Cochrane Database Syst. Rev. 3, CD000510 (2012).

71. Soll, R. F. Synthetic surfactant for respiratory distress syndrome in preterm infants. Cochrane Database Syst. Rev. 2, CD001149 (2000).

72. Natarajan, C. K. et al. Surfactant therapy and antibiotics in neonates with meconium aspiration syndrome: a systematic review and meta-analysis. J. Perinatol. 36(Suppl 1), 49-54 (2016).

73. Amizuka, T., Shimizu, H., Niida, Y. \& Ogawa, Y. Surfactant therapy in neonates with respiratory failure due to haemorrhagic pulmonary oedema. Eur. J. Pediatr. 162, 697-702 (2003).

74. Nishina, K. et al. Effects of exogenous surfactant on acute lung injury induced by intratracheal instillation of infant formula or human breast milk in rabbits. Anesthesiology 91, 240-252 (1999).

75. De Luca, D. et al. Bile acids cause secretory phospholipase A2 activity enhancement, revertible by exogenous surfactant administration. Intensive Care Med. 35, 321-326 (2009).

76. Wang, Z. \& Notter, R. H. Additivity of protein and nonprotein inhibitors of lung surfactant activity. Am. J. Respir. Crit. Care Med. 158, 28-35 (1998).

77. Hahn, S., Choi, H. J., Soll, R. \& Dargaville, P. A. Lung lavage for meconium aspiration syndrome in newborn infants. Cochrane Database Syst. Rev. 4, CD003486 (2013).

78. Aziz, A. \& Ohlsson, A. Surfactant for pulmonary haemorrhage in neonates. Cochrane Database Syst. Rev. 7, CD005254 (2012).

79. Been, J. V. et al. Chorioamnionitis alters the response to surfactant in preterm infants. J. Pediatr. 156, 10-15.e1 (2010).

80. Herting, E. et al. Surfactant treatment of neonates with respiratory failure and group B streptococcal infection. Members of the Collaborative European Multicenter Study Group. Pediatrics 106, 957-6 (2000). 
Surfactant replacement therapy: from biological basis to current clinical... $\mathrm{R}$ Hentschel et al.

81. Björklund, L. J. et al. Manual ventilation with a few large breaths at birth compromises the therapeutic effect of subsequent surfactant replacement in immature lambs. Pediatr. Res. 42, 348-355 (1997).

82. van Kaam, A. H. et al. Response to exogenous surfactant is different during open lung and conventional ventilation. Crit. Care Med. 32, 774-780 (2004).

83. Moriette, G. et al. Prospective randomized multicenter comparison of highfrequency oscillatory ventilation and conventional ventilation in preterm infants of less than 30 weeks with respiratory distress syndrome. Pediatrics 107, 363-372 (2001).
84. Goldsmith, L. S. et al. Immediate improvement in lung volume after exogenous surfactant: alveolar recruitment versus increased distention. J. Pediatr. 119, 424-428 (1991).

85. Anderson, J. C., Molthen, R. C., Dawson, C. A. \& Haworth, S. T. Effect of ventilation rate on instilled surfactant distribution in the pulmonary airways of rats. J. Appl. Physiol. 97, 45-56 (2004).

86. Miedema, M. et al. Changes in lung volume and ventilation during surfactant treatment in ventilated preterm infants. Am. J. Respir. Crit. Care Med. 184, 100-105 (2011). 